\title{
ORIGINAL ARTICLE Isolation-by-distance in landscapes: considerations for landscape genetics
}

\author{
MJ van Strien ${ }^{1,2}$, R Holderegger ${ }^{2,3}$ and HJ Van Heck $^{4,5}$
}

In landscape genetics, isolation-by-distance (IBD) is regarded as a baseline pattern that is obtained without additional effects of landscape elements on gene flow. However, the configuration of suitable habitat patches determines deme topology, which in turn should affect rates of gene flow. IBD patterns can be characterized either by monotonically increasing pairwise genetic differentiation (for example, $\mathrm{F}_{\mathrm{ST}}$ ) with increasing interdeme geographic distance (case-I pattern) or by monotonically increasing pairwise genetic differentiation up to a certain geographical distance beyond which no correlation is detectable anymore (caseIV pattern). We investigated if landscape configuration influenced the rate at which a case-IV pattern changed to a case-I pattern. We also determined at what interdeme distance the highest correlation was measured between genetic differentiation and geographic distance and whether this distance corresponded to the maximum migration distance. We set up a population genetic simulation study and assessed the development of IBD patterns for several habitat configurations and maximum migration distances. We show that the rate and likelihood of the transition of case-IV to case-I F ST $_{\text {-distance relationships was }}$ strongly influenced by habitat configuration and maximum migration distance. We also found that the maximum correlation between genetic differentiation and geographic distance was not related to the maximum migration distance and was measured across all deme pairs in a case-I pattern and, for a case-IV pattern, at the distance where the $\mathrm{F}_{\mathrm{ST}}$-distance curve flattens out. We argue that in landscape genetics, separate analyses should be performed to either assess IBD or the landscape effects on gene flow.

Heredity (2015) 114, 27-37; doi:10.1038/hdy.2014.62; published online 23 July 2014

\section{INTRODUCTION}

Ever since Wright (1943) described isolation-by-distance (IBD), patterns of spatial genetic structure have been extensively studied in population genetic simulation models (Epperson, 2003; Epperson et al., 2010) and in natural populations (Crispo and Hendry, 2005; Jenkins et al., 2010; Storfer et al., 2010). In most of these studies, migration probability is a function of geographic straight-line distance. Recently, landscape genetic studies have incorporated more complex landscape measures than straight-line distance aiming to give a more realistic estimate of the effective distance between demes (Holderegger and Wagner, 2006). In general, the variation in estimates of pairwise genetic distances explained by these effective distances is compared with that explained by geographic distances alone (that is, IBD). The latter is regarded as the most simple landscape genetic pattern that would be obtained even if there were no landscape effects and migration was thus only constrained by distance between demes (Spear et al., 2005; Balkenhol et al., 2009; Jenkins et al., 2010). This notion may have originated from spatially explicit simulation studies of IBD patterns, in which demes or individuals are usually placed in regular lattices throughout homogeneous spaces (Guillot et al., 2009; Epperson et al., 2010). Indeed, distance-constrained migration in such models produces IBD patterns that are not influenced by any landscape elements. However, a heterogeneous landscape will not only affect migration probabilities between demes, but also the spatial arrangement of demes (that is, deme topology). Only few studies have examined the effect of deme topology on patterns of IBD. Doligez et al. (1998) concluded that strong spatial clumping of individuals leads to slight increases in spatial genetic autocorrelation, and Ezard and Travis (2006) found fixation time to be greater in long and narrow habitats. These simulation studies thus suggest that the spatial arrangement of habitat patches has an influence on genetic patterns in general and IBD patterns in specific. Although Robledo-Arnuncio and Rousset (2010) found that effective population density $\left(D_{e}\right)$ and effective dispersal rate $\left(\sigma_{e}^{2}\right)$ 'depend in a complex way on the spatial and temporal demographic heterogeneities of the population, in equilibrium situations, their product $\left(D_{e} \sigma_{e}^{2}\right)$ was still related to the slope of the correlation between genetic distance (that is, $\mathrm{F}_{\mathrm{ST}} /(1-$ $\left.\mathrm{F}_{\mathrm{ST}}\right)$ ) and log-transformed geographic distance between demes or individuals. However, landscape geneticists usually do not know whether the genetic data sampled from natural populations reflect an equilibrium or non-equilibrium state. In the present study, we therefore assess to what degree habitat configuration influences equilibrium and non-equilibrium IBD patterns. We abandon the regular lattice setup of demes or individuals, as commonly used in population genetic simulation studies (Doligez et al., 1998; Epperson et al., 2010), and instead use irregular habitat configurations and deme topologies, which better reflect the natural study systems typically used in landscape genetics.

${ }^{1}$ Planning of Landscape and Urban Systems, ETH Zurich, Stefano-Franscini-Platz 5, Zurich, Switzerland; ${ }^{2}$ WSL Swiss Federal Research Institute, Zürcherstrasse 111, Birmensdorf, Switzerland; ${ }^{3}$ Department of Environmental System Sciences, ETH Zurich, Universitätsstrasse 16, Zurich, Switzerland; ${ }^{4}$ Earth and Ocean Sciences, Cardiff University, Main Building, Park Place, Cardiff, UK and 5 Institute of Earth Sciences, Utrecht University, Budapestlaan 4, Utrecht, The Netherlands

Correspondence: Dr MJ van Strien, Planning of Landscape and Urban Systems, ETH Zurich, Stefano-Franscini-Platz 5, IRL-HIL H 42.3, Zurich, ZH CH-8093, Switzerland. E-mail: maarten@vstrien.nl

Received 15 October 2013; revised 13 May 2014; accepted 27 May 2014; published online 23 July 2014 
Hutchison and Templeton (1999) described hypothetical and empirical IBD patterns and showed that an $\mathrm{F}_{\mathrm{ST}}$-distance relationships are not always of 'case-I' type, which is characterized by monotonically increasing pairwise $\mathrm{F}_{\mathrm{ST}}$ values with increasing interdeme distance due to an equilibrium of gene flow and random genetic drift (Figure 1; Hutchison and Templeton, 1999). Namely, beyond a certain interdeme distance, gene flow (including indirect gene flow over several generations) can be so limited compared with genetic drift or mutation that there is no significant $\mathrm{F}_{\mathrm{ST}}$-distance slope anymore. The corresponding 'case-IV' type $\mathrm{F}_{\mathrm{ST}}$-distance relationship is characterized by monotonically increasing $\mathrm{F}_{\mathrm{ST}}$ values up to a certain distance, beyond which the plot flattens out and $\mathrm{F}_{\mathrm{ST}}$ values cease to increase (Figure 1; Hutchison and Templeton, 1999). Because Hutchison and Templeton's example plots and description of potential $\mathrm{F}_{\mathrm{ST}}-$ distance relationships are simple and intuitive references, we adopt their 'caseI' and 'case-IV' terminology in the present study (Figure 1). The caseIV situation described by Hutchison and Templeton (1999) is a transitional state between a situation of panmixia and a case-I situation, which implies that case-IV patterns represent non-equilibrium states, whereas case-I patterns refer to equilibrium states. Such non-equilibrium case-IV situations should not be confused with equilibrium states of $\mathrm{F}_{\mathrm{ST}}$-distance relationships that exhibit an asymptotic curve, which can resemble a case-IV IBD pattern (Rousset, 1997). Hutchison and Templeton (1999) explained regional differences in IBD patterns with time-since-colonization and the (historical) presence of barriers (that is, forests). Their results have, however, not been discussed in the light of differences in habitat configuration between study regions. Therefore, the first goal of the present study is to determine if habitat configuration has an effect on the rate at which IBD patterns change from case-IV to case-I.

Most landscape genetic studies measure the level of IBD by quantifying the linear correlation between genetic (for example, $\mathrm{F}_{\mathrm{ST}}$ ) and geographic distances from all deme pairs in a data set (Crispo and Hendry, 2005; Balkenhol et al., 2009; Jenkins et al., 2010). However, to highlight dispersal barriers due to landscape elements, several authors have recently argued that landscape genetic analyses should be restricted to only those pairs of demes between which direct gene flow is possible (Angelone et al., 2011; Keller et al., 2013). As most landscape genetic studies make use of historical gene flow measures (for example, $\mathrm{F}_{\mathrm{ST}}$; Jenkins et al., 2010; Storfer et al., 2010), gene flow is not only measured between deme pairs that are within each other's maximum migration distance, but also between demes beyond this distance that experience indirect gene flow (over several
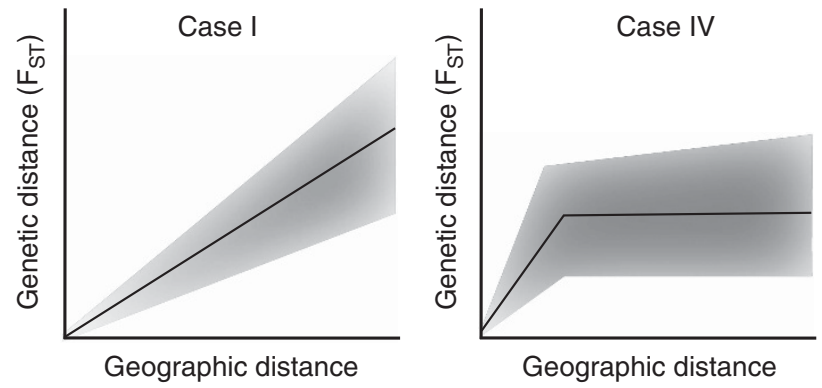

Figure 1 Two hypothetical $\mathrm{F}_{\mathrm{ST}}$-distance relationships modified from Hutchison and Templeton (1999). The case-I relationship (left) is characterized by a monotonically increasing $\mathrm{F}_{\mathrm{ST}}$ and scatter with interdeme geographic distance. The same can be observed in a case-IV relationship (right) up to a certain geographic distance, after which no relationship between F $_{\text {ST }}$ and interdeme distance is detectable anymore. generations) via intermediate demes. However, the inhibiting or facilitating effect on movement of certain landscape elements can only be detected between those demes that potentially exchange migrants (that is, deme pairs separated by a distance lower than the maximum migration distance). If there is no possibility for demes to exchange direct migrants (that is, their interdeme geographical distance is larger than the maximum migration distance), the lack of gene flow is due to physical limitations of the focal species and not necessarily caused by any landscape effect on migration. For landscape genetics, it is thus important to differentiate between these two types of limitations. Indeed, in a landscape genetic analysis on a grasshopper species Keller et al. (2013) found that, compared with considering all deme pairs, a much higher model fit and a better distinguishability of the most likely migration habitat was obtained when only deme pairs separated up to $3 \mathrm{~km}$ were considered. This distance threshold corresponded to the distance at which the $\mathrm{F}_{\mathrm{ST}}-$ distance plot flattened out in a case-IV IBD pattern (Keller et al., 2013), but it also corresponded to the estimated maximum migration distance of this grasshopper (van Strien et al., 2014). Theoretical population genetic studies have shown that the distance at which an $\mathrm{F}_{\mathrm{ST}}$-distance plot flattens out can be larger than the maximum migration distance in equilibrium (that is, asymptotic case- $\mathrm{F}_{\mathrm{ST}}-$ distance relationship) and non-equilibrium situations (Slatkin, 1993). However, for the analysis of IBD in landscape genetics, it has yet to be determined at what interdeme distance the highest correlation between $\mathrm{F}_{\mathrm{ST}}$ and interdeme geographic distance can be measured and whether this distance is determined by the maximum migration distance of a given species. If the highest $\mathrm{F}_{\mathrm{ST}}$-distance correlation is measured across all deme pairs separated by distances up to the maximum migration distance, then the distance threshold of an IBD analysis corresponds to the recommended threshold for the detection of landscape effects on gene flow (that is, maximum migration distance). However, if the highest correlation value is measured, for a case-IV pattern, at the distance where the $\mathrm{F}_{\mathrm{ST}}$-distance curve flattens out and, for a case-I pattern, across all deme pairs, there is a discrepancy between the two thresholds. The second goal of this study is thus to determine at what interdeme distance the highest $\mathrm{F}_{\mathrm{ST}}$ distance correlation is measured.

To address the above two study goals, we set up an agent-based population genetic simulation model. For various habitat configurations and maximum migration distances (MMD), we examined the development of IBD patterns, determined whether these patterns could best be described as case-I or case-IV $\mathrm{F}_{\mathrm{ST}}$-distance relationships and at what interdeme distance the highest $\mathrm{F}_{\mathrm{ST}}$-distance correlation was measured. In contrast to other population genetic simulation studies that have focussed on IBD patterns from a theoretical point of view (Epperson, 2003; Epperson et al., 2010), we specifically designed a simulation model that accommodated current landscape genetic practice (Balkenhol et al., 2009; Jenkins et al., 2010; Storfer et al., 2010), so that our findings can easily be integrated into future landscape genetic studies. For instance, we do not assess IBD patterns with Moran's I correlograms, as often done in population genetics (Epperson, 2003), but use linear correlations between $\mathrm{F}_{\mathrm{ST}}$ and geographic distance instead, as is done in landscape genetics (Jenkins et al., 2010). Also, the regular lattice setup of landscapes is abandoned, and replaced with habitat configurations that better reflect natural landscapes.

\section{MATERIALS AND METHODS}

We developed a stochastic agent-based numerical model to simulate genetic differentiation between demes that were placed in the habitat cells of 
two-dimensional landscape grids. Diploid individuals (agents) were allowed to migrate between demes. Migration probabilities between demes were drawn from Gaussian or exponential migration functions (see below). After a certain number of non-overlapping migration-reproduction cycles (that is, generations), we extracted matrices of pairwise $\mathrm{F}_{\mathrm{ST}}$ and geographic distances, which were post processed to determine IBD patterns. Genetic patterns in population genetic studies have traditionally been simulated with either discrete demes (that is, stepping-stone models; Kimura and Weiss, 1964) or a more or less continuous distribution of individuals (Wright, 1943; Guillot et al., 2009; Epperson et al., 2010). We chose to structure our individuals in discrete demes, because in more than $80 \%$ of landscape genetic studies, individuals were sampled from demes and, subsequently, genetic differentiation was determined between demes (Storfer et al., 2010). We anticipate that this indicates that most landscape genetic studies focus on species that occur in discrete demes. Mutation was not considered as a source of genetic variation. The numerical code, named Concordia, was written in MATLAB (The MathWorks, Natick, MA, USA) and is available online (Dryad data repository).

To create different configurations of habitats, we generated neutral landscapes, which are frequently used in landscape ecology to test hypotheses about habitat configuration and fragmentation on ecological processes (Gardner and Urban, 2007). Neutral landscapes have also been used in landscape genetics (Ezard and Travis, 2006). With the programme QRULE 4.2 (Gardner and Urban, 2007), we generated five binary habitat-matrix landscapes of $128 \times 128$ cells, of which $50 \%$ were classified as habitat and $50 \%$ as matrix ( \pm max. $0.51 \%$; Figure 2). This resulted in landscapes with $\sim 8192$ habitat cells. The level of habitat fragmentation (measured with a spatial autocorrelation parameter $\mathrm{H})$ was the same in all landscapes $(H=0.5$; 'next nearest neighbourhood rule' setting in QRULE). The five neutral landscapes were finite; that is, they had edges. Distance units in the present study were expressed as number of cells on the landscape grid.

At the beginning of each simulation, we randomly placed 100 equally sized demes in habitat cells of a neutral landscape. The total density of demes within habitat and within the total landscape was equal in all simulations. The locations of demes remained constant during the course of a simulation. We chose to randomly place demes within the habitat, because we wanted to determine if certain habitat configurations consistently produce a certain type of IBD, regardless of random deme topology. Furthermore, in natural circumstances, species may be bound to a certain habitat type (for example, forests, grasslands, wetlands), but within these habitats exhibit a non-regular deme topology due to, for instance, microclimatic heterogeneity (Corney et al., 2004), competition (Meineri et al., 2012) or stochastic processes (Hubbell, 2001). Each deme consisted of 50 diploid individuals, which were characterized by their genotypes at 10 neutral bi-allelic loci. At the beginning of a simulation, genotypes were defined by randomly allocating 2 out of 10 alleles to each individual's loci, simulating an initial state of panmixia.

The first step in each migration-reproduction cycle was the migration of individuals from their natal deme to other demes in the landscape. For a broad range of species, mark-recapture or trapping studies have shown that a large proportion of individuals is sedentary (or philopatric) and will not disperse far from their location of birth, whereas a smaller group of individuals is rather vagile and migrates much further from the natal location (Paradis et al., 1998; Chapman et al., 2011). We simulated such migration behaviour by, at the beginning of each migration-reproduction cycle, selecting individuals that would leave their natal deme. Each individual had a probability of 0.2 of being selected, so that, on average, $20 \%$ of deme members emigrated from their natal deme. The remaining $\sim 80 \%$ were sedentary individuals, which remained in their natal deme and became, together with new immigrants, the parents of the next generation. Each migrating individual had a certain probability to reach other demes as dictated by a probability density function, which determined the migration probability over a certain geographic distance. As we implemented no difference between migration probabilities through habitat or matrix, migration between demes was purely a function of geographic distance. The sum of the probabilities of migration from a natal deme to other demes could be lower than 1 , meaning that some migrants never reached a new deme. These migrants were removed from the simulation before reproduction. Note that by applying this two-phase approach of first selecting migrants and only then selecting their destinations, we ensured both a statistically stable emigration rate for all demes and an immigration probability that was only dictated by a distance-dependent migration function, which is in accordance with the metapopulation dynamics theory described by Hanski (1998), who stated that 'because it is reasonable to assume that mortality within the habitat patches does not depend on isolation, unlike mortality during migration, one can tease apart, at least in principle, the two kinds of mortality'. For every random deme topology, we also checked that the sum of the probabilities of migration from a natal deme to other demes never exceeded 1.

Two types of probability distribution functions (pdf) were used to simulate the migration probability over a certain distance between demes. We used exponential and Gaussian migration functions. Two-dimensional pdfs are used

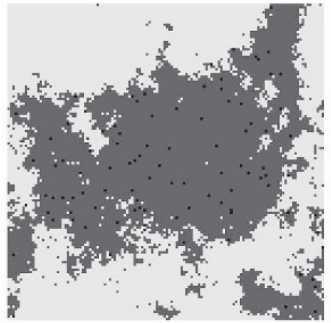

Landscape A

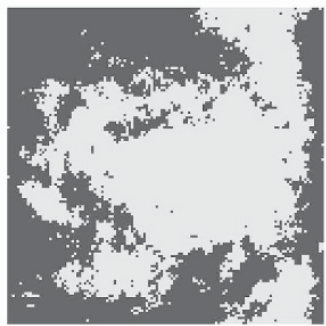

Landscape D

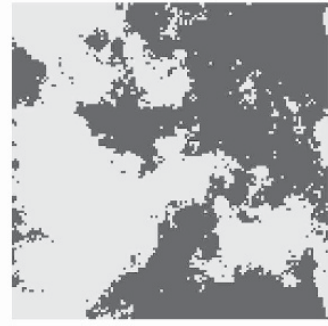

Landscape B

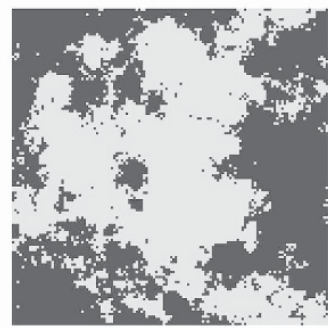

Landscape $\mathrm{E}$

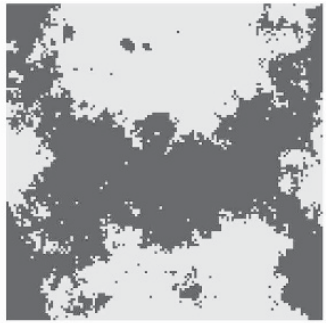

Landscape C

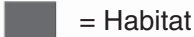

= Matrix

Figure 2 The five neutral landscapes that were used in the present population genetic simulation study. Simulated demes were randomly placed in habitat cells. As an example, we show a random topology of 100 demes in landscape A (black cells represent demes). All landscapes have $50 \%$ habitat and $50 \%$ matrix, the same level of fragmentation $(H=0.5)$ and dimensions of $128 \times 128$ cells. 
if the migration is simulated in two-dimensional landscapes (Austerlitz et al., 2004). However, preliminary tests of our simulations showed that gene flow with two-dimensional pdfs was generally too low for IBD to emerge. Therefore, we chose to use one-dimensional pdfs, which resulted in a range of immigration rates per generation (see Results) that were comparable to the range typically observed in natural populations (that is, $\sim 0-10 \%$; Bowne and Bowers, 2004). For the sake of clarity, we characterize the different probability functions by a maximum migration distance. We define maximum migration distance as the distance at which probability of migration equalled 0.0001 . Thus, we did not set an absolute maximum migration distance, but a distance above which migration is unlikely. This is analogous to natural situations where there is no absolute maximum migration distance and where it is probable that there is variability in the movement capabilities of individuals. For the Gaussian pdf, we experimented with variances of 15, 40, 110 and 200, resulting in MMD of 14.4, 22.7, 36.1 and 47.4 distance units. For the exponential pdf, we experimented with $\mu$-values of $2,3,4$ and 5, which resulted in MMD of 17.0, 24.3, 31.2 and 38.0 distance units.

After the migration step, random mating took place within each deme. Within demes, each individual was randomly paired with another individual to form mating pairs that produced 10 diploid offspring. In case of an uneven number of deme members (resulting from an uneven number of emigrants and immigrants), one random individual did not mate. Mating was thus sexual, in the sense that it was always between two individuals, resulting in Mendelian inheritance. From all offspring in a deme, 50 individuals were randomly drawn to reach maturity and form the next generation of parents, thereby maintaining a constant deme size at the beginning of each cycle. At this point, the end of a migration-reproduction cycle was reached and the next cycle started. Each simulation was stopped after 500 such cycles.

For each combination of the five landscapes and the eight MMD values (that is, four for the Gaussian pdf and four for the exponential pdf), we performed 50 replicated simulations (that is, 50 times a new random topology of 100 demes followed by 500 migration-reproduction cycles). For each simulation, we analysed the genetic differentiation between demes after 50 and 500 migration-reproduction cycles (that is, generations). Genetic differentiation was quantified by $\mathrm{F}_{\mathrm{ST}}$, because its formulation is based on two focal microevolutionary processes (that is, gene flow and genetic drift) relevant to
IBD patterns and because it is the most frequently used estimates of genetic distance in landscape genetic studies (Jenkins et al., 2010; Storfer et al., 2010). Multiallelic pairwise $\mathrm{F}_{\mathrm{ST}}$ values were calculated following Nei (1977). Hutchison and Templeton (1999) used $\mathrm{F}_{\mathrm{ST}}$ values calculated with Weir and Cockerham's (1984) approach (Goudet, 1995). For our analyses, an unbiased estimator of genetic differentiation was not necessary, as we have maintained a fixed number of demes and a fixed deme size throughout our simulations. Furthermore, pairwise estimates of such estimates of historical gene flow have shown to be highly correlated (Van Strien et al., 2012; Keller et al., 2013). We therefore assume that the differences in calculation of both estimates had a negligible effect.

Subsequently, we determined the type of $\mathrm{F}_{\mathrm{ST}}$-distance correlation (that is, case-I or case-IV) and the interdeme distance at which the $\mathrm{F}_{\mathrm{ST}}$-distance correlation was highest with an approach, in which we created groups of deme pairs and determine the $\mathrm{F}_{\mathrm{ST}}$-distance correlations for each group. These groups were defined by selecting all deme pairs that were separated by 0 to $d$ distance units. $d$ was increased from 1.81-181 distance units in intervals of 1.8 (that is, 99 values of $d$ ). A $d$ of 181 represented the full diagonal length of the landscape, which resulted in all deme pairs being selected. To prevent biased correlation estimates due to small sample size (Montgomery and Morrison, 1973), we only considered $d$ values that resulted in groups of 50 or more deme pairs. To quantify the linear $\mathrm{F}_{\mathrm{ST}}$-distance correlation in landscape genetics, the Mantel $r$ correlation coefficient is usually used (Storfer et al., 2010). We thus determined the value of $d$ at which Mantel $r$ between $\mathrm{F}_{\mathrm{ST}}$ and geographic distance was maximized and referred to this $d$ as the distance of maximum correlation (DMC; Figure 3). We correlated $\mathrm{F}_{\mathrm{ST}}$ to untransformed geographic distances and $\mathrm{F}_{\mathrm{ST}} /\left(1-\mathrm{F}_{\mathrm{ST}}\right)$ to log-transformed geographic distances. The latter transformation is often used in landscape genetics following Rousset (1997). Post processing of $\mathrm{F}_{\mathrm{ST}}$ values was performed in $\mathrm{R}$ (R Development Core Team, 2012).

The distribution of DMC values was displayed as bean plots (Kampstra, 2008), which are analogous to mirrored, smoothed vertical histograms. These plots are suitable for determining whether an $\mathrm{F}_{\mathrm{ST}}$-distance correlation was of case-I or case-IV type (see Results section). Furthermore, we displayed the maximum $\mathrm{F}_{\mathrm{ST}}$-distance correlation (that is, correlation at DMC) in relation to the correlation that was obtained across all deme pairs.

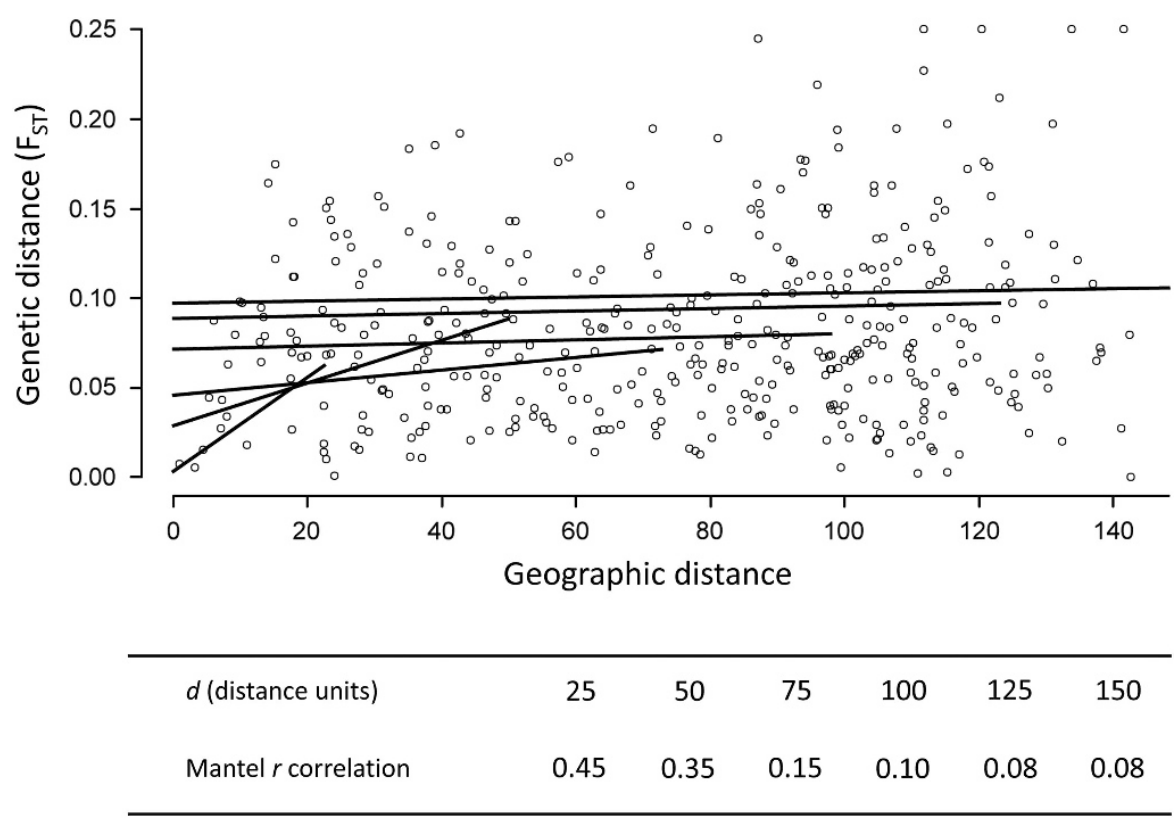

Figure 3 Demonstration of how the distance of DMC was calculated. From plots of pairwise genetic $\left(\mathrm{F}_{\mathrm{ST}}\right)$ and geographic distances, we selected deme pairs with an interdeme distance up to a maximum distance $d$. For each selection of deme pairs, we then calculated the Mantel $r$ correlation coefficient between geographic distance and $\mathrm{F}_{\mathrm{ST}}$. Correlation trend lines belonging to different $d$ values are shown in black. In the table, we show the correlation coefficients calculated for a series of $d$-values. In the present example, DMC would be 25 , as $d=25$ resulted in the highest $\mathrm{F}_{\mathrm{ST}}$-distance correlation (that is, Mantel $r$ correlation $=0.45$ ). 


\section{RESULTS}

The five neutral landscapes generated were labelled A-E (Figure 2). In landscape A, there was a large central area of habitat, surrounded by matrix, whereas in landscapes D and E, the habitat was distributed around a central patch of matrix (Figure 2). Thus in landscape A, most demes were clustered together in the central habitat, whereas in landscapes D and E, most demes were scattered on the periphery of the study area. These differences in deme topology were reflected by the differences in mean geographic distance between the 100 demes randomly placed in habitat (median of the average distances: landscape $A=55.6$; landscape $B=62.2$; landscape $C=63.0$, landscape $D=73.8$; landscape $E=74.9$ ). The distribution of mean distances was largely overlapping between landscape B and $\mathrm{C}$ as well as D and E (Supplementary Figure 1).

We obtained similar results for simulations with Gaussian and exponential migration functions. For the sake of clarity, we only discuss the results from simulations with the Gaussian migration function here. However, results from simulations with the exponential pdf are given in the Supplementary Material (Supplementary Figures 2-4).

Although emigration probability was fixed for each individual (that is, 0.2 ), the immigration probability of a migrant was dependent on geographic distance (see Methods section) and, thus, the differences in mean geographic distances between all demes in the five neutral landscapes also resulted in differences in the average immigration probability of a migrant. For instance, with the Gaussian migration function and $\mathrm{MMD}=14.4$, the average probabilities that a migrant reached another deme (that is, immigration probability) in landscapes $\mathrm{A}, \mathrm{B}, \mathrm{C}, \mathrm{D}$ and $\mathrm{E}$ were $0.0938,0.0922,0.0938,0.0871$ and 0.0838 , respectively, and for $\mathrm{MMD}=47.4$, these immigration probabilities changed to $0.2721,0.2398,0.2508,0.2075$ and 0.2014 , respectively.

The interdeme distance of DMC was measured at generations 50 and 500 in each simulation. DMC distributions from correlations between $\mathrm{F}_{\mathrm{ST}}$ and untransformed geographic distances were similar to the DMC distributions from correlations between $\mathrm{F}_{\mathrm{ST}} /\left(1-\mathrm{F}_{\mathrm{ST}}\right)$ and log-transformed geographic distances (Figure 4 and Supplementary Figure 5). We therefore chose to only show the results from untransformed geographic distance measures (Figure 4). Examining the distributions of DMC values, we distinguished two types of unimodal distributions. First we observed a unimodal distribution with a peak of DMC values just below or slightly above MMD (Figures $4 \mathrm{a}-\mathrm{c}$ and landscapes $\mathrm{D}$ and $\mathrm{E}$ in Figure $4 \mathrm{~d}$ ). The second unimodal DMC distribution (landscapes A, B and C in Figures $4 \mathrm{f}-\mathrm{h}$ ) peaked between 140 and 170 distance units. The bimodal distribution of DMC values that was visible in some cases (for example, landscapes $\mathrm{A}, \mathrm{B}$ and $\mathrm{C}$ in Figure 4d), was a combination of both unimodal distributions.

Next we examined what kind of $\mathrm{F}_{\mathrm{ST}}$-distance plot resulted in the two unimodal distributions of DMC values. We regarded the DMC distribution of landscape $\mathrm{E}$ at generation 500 and $\mathrm{MMD}=36.1$ (Figure 4f) as characteristic for the first type of unimodal distribution (that is, peak of DMC values just below or above MMD) and that of landscape A with the same settings (Figure 4f) as characteristic for the second unimodal distribution (that is, peak of DMC between 140 and 170 distance units). For both these distributions, we created $\mathrm{F}_{\mathrm{ST}^{-}}$ distance plots by averaging the median and lower and upper bounds of the scatter of $\mathrm{F}_{\mathrm{ST}}$-distance plots from 50 random topologies of 100 demes (Figure 5). The first type of DMC distributions resulted in a monotonic increase of $\mathrm{F}_{\mathrm{ST}}$ and scatter up to a certain geographic distance, beyond which the plot flattened out and lost its correlative character (Figure 5a). The distance at which the plot flattened coincided with the peak of the first type of DMC distribution (that is, at $\sim 68$ distance units; Figures $4 \mathrm{f}$ and $5 \mathrm{a}$ ). We referred to this $\mathrm{F}_{\mathrm{ST}}-$ distance relationship as a case-IV type (Hutchison and Templeton, 1999). The second type of DMC distribution resulted in monotonically increasing $\mathrm{F}_{\mathrm{ST}}$ values and scatter with increasing geographic distance up to the maximum (diagonal) length of the study area (that is, between 128 and 180 distance units; Figure 5b). We termed this type of $\mathrm{F}_{\mathrm{ST}}$-distance relationship a case-I type (Hutchison and Templeton, 1999). For both plots, we observed a slightly increasing slope of median $\mathrm{F}_{\mathrm{ST}}$ values at larger geographic distances (Figure 5).

The configuration of habitat in the landscape had a strong effect on the type of IBD pattern (that is, case-I or case-IV). In some landscapes only case-I or case-IV distributions were observed for a certain MMD and generation. For instance, with $\mathrm{MMD}=36.1$ (Figures $4 \mathrm{e}$ and $\mathrm{f}$ ), the random deme topology in habitat of landscape A produced mainly case-I $\mathrm{F}_{\mathrm{ST}}$-distance relationships at generations 50 and 500, whereas in landscape E, predominantly case-IV relationships were observed for both generations. Landscapes where both case-I and case-IV relationships were found for a certain MMD and generation (landscapes B and C in Figures $4 \mathrm{~d}$ and e) indicated that not only habitat configuration, but also deme topology in the habitat had an effect on IBD patterns. Landscapes in which mean geographic distance between all deme pairs was similar (that is, landscapes B and C as well as D and E; Supplementary Figure 1) also showed similar patterns of DMC distributions (Figure 4). An exception were the differences in DMC distributions of landscape $\mathrm{D}$ and $\mathrm{E}$ for $\mathrm{MMD}=36.1$ at generation 500 (Figure $4 \mathrm{f}$ ) and $\mathrm{MMD}=47.4$ at generation 50 (Figure $4 \mathrm{~g}$ ).

The distance at which the $\mathrm{F}_{\mathrm{ST}}$-distance plot flattened out in case-IV relationships (that is, peak of the DMC distribution) could not be predicted from MMD. In some cases, the DMC distribution peaked below the MMD (Figures $4 \mathrm{a}$ and c), whereas in other cases, the peak was located above the MMD (landscapes D and E in Figures $4 \mathrm{~b}$, d and e). No consistency in the difference in distance between the MMD and the peak of the DMC distribution could be found between landscapes with similar mean geographic distances between demes (for example, landscapes B and C as well as D and E in Figure $4 \mathrm{~d}$ ). Furthermore, for case-I relationships, the $\mathrm{F}_{\mathrm{ST}}$ values kept on monotonically increasing far beyond the MMD (for example, landscapes A, $B$ and $C$ in Figure 4h).

MMD had a clear effect on the rate at which case-I $F_{S T}$-distance relationships appeared for certain landscapes. For instance, in landscape $\mathrm{A}$ at generation 50 (Figures $4 \mathrm{a}, \mathrm{c}$, e and g) there were hardly any simulations that resulted in case-I relationships with $\mathrm{MMD}=14.4$ or $\mathrm{MMD}=22.7$, whereas practically all relationships were case-I type with $\mathrm{MMD}=36.1$ or $\mathrm{MMD}=47.4$. We observed that case-IV relationships at generation 50 either evolved to a case-I relationship over time or remained in a case-IV state for more than 450 generations. For an MMD of 14.4 (Figures $4 \mathrm{a}$ and b), the vast majority of simulations resulted in case-IV $\mathrm{F}_{\mathrm{ST}}$-distance relationships at generations 50 and 500. A similar pattern was observed in landscape $\mathrm{E}$ with $\mathrm{MMD}=36.1$ (Figures $4 \mathrm{e}$ and $\mathrm{f}$ ), where the peak of the DMC only slightly increased after 450 generation. A case-IV changing to a case-I relationship could be observed in the DMC distribution of landscape A at MMD $=22.7$ (Figures $4 \mathrm{c}$ and $\mathrm{d}$ ).

The $\mathrm{F}_{\mathrm{ST}}$-distance correlation coefficients calculated for all deme pairs were different from the maximum correlation coefficients (that is, the correlation at DMC; Figure 6). For case-IV relationships, negative $\mathrm{F}_{\mathrm{ST}}$-distance correlations were measured between all deme pairs for a certain MMD and landscape, whereas the maximum correlation was always positive (Figures $6 \mathrm{a}$ and c). It is noteworthy 

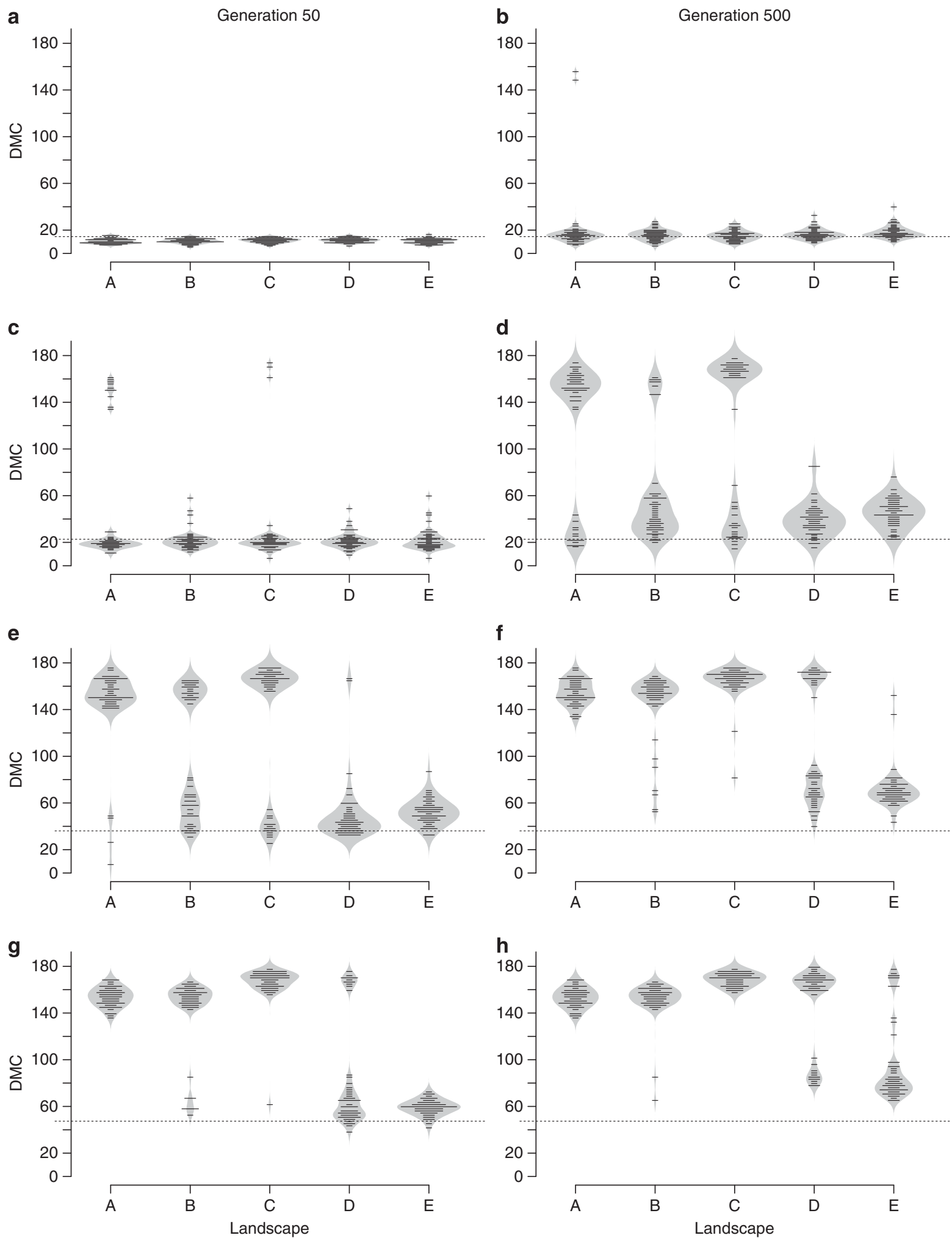

Figure 4 Bean plots showing the distance of DMC for five different neutral landscapes (Figure 2) and a range of MMD at generations 50 (a, c, e, $\mathbf{g}$ ) and 500 $(\mathbf{b}, \mathbf{d}, \mathbf{f}, \mathbf{h})$. Gaussian migration probability functions were used in these simulations. The length of the black lines in a bean plot indicates the number of times a certain DMC was scored from the 50 random topologies of 100 demes. Similarly, the grey areas of the bean plot show the smoothed frequency distributions of observations at different DMC values, analogous to a mirrored histogram. The dashed lines indicate the MMD, at which the migration probability from a Gaussian migration function with a certain variance equalled 0.0001. We tested four MMDs: 14.4 (a, b), 22.7 (c, d), 36.1 (e, f) and 47.4 (g, h). A peak in

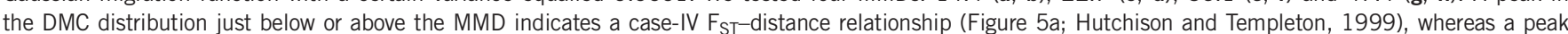
in the DMC distribution between 140 and 170 distance units indicates a case-I relationship (Figure 5b; Hutchison and Templeton, 1999). 
a

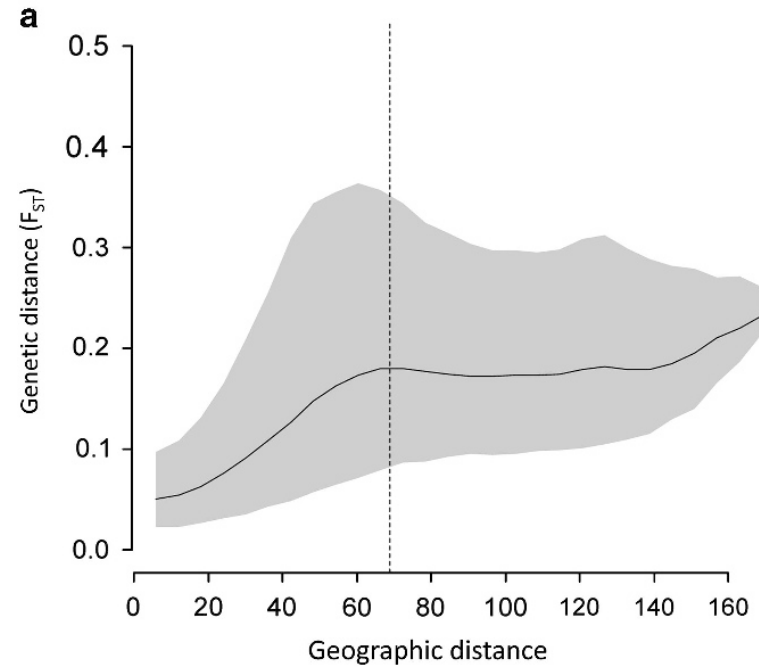

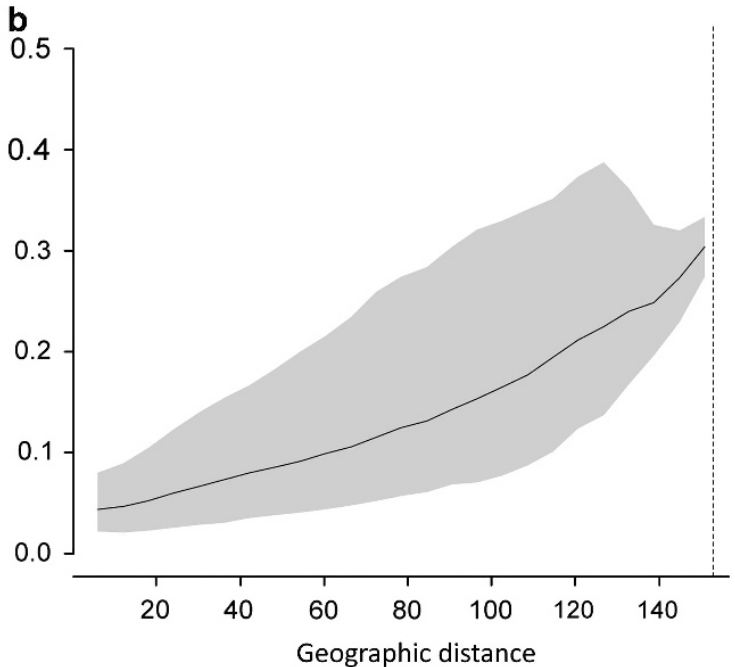

Figure 5 Two examples of genetic and geographic distance plots obtained after 500 generations. Gaussian migration probability functions were used. The displayed median (black line) and lower and upper bounds of the scatter (grey area) of the $\mathrm{F}_{\mathrm{ST}}$ values are the result of averaging the median and lower and upper bounds of $\mathrm{F}_{\mathrm{ST}}$-distance plots from 50 random topologies of 100 demes. The dashed line indicates the median distance of maximum $\mathrm{F}_{\mathrm{ST}}$-distance correlation (that is, the peak of the DMC distribution; Figure 4). Both plots are based on the results of simulations that used a maximum migration distance of 36.1 (Figure 4f). The left plot (a) gives the results of the simulations on landscape $E$ (Figure 2 ) and is similar to a case-IV $F_{S T}$-distance relationship from Hutchison and Templeton (1999); Figure 1. The right plot (b) presents the results of simulations on landscape A (Figure 2) and represents the case-I $\mathrm{F}_{\mathrm{ST}}-$ distance relationship from Hutchison and Templeton (1999); Figure 1. Relatively few deme pairs have a high interdeme geographic distance, which explains the reduced scatter at high distance values.

that the range of maximum correlation coefficients for a certain MMD at a certain generation showed a strong overlap between landscapes (Figure 6), regardless of the type of $\mathrm{F}_{\mathrm{ST}}$-distance relationship. For instance, at generation 500 and $\mathrm{MMD}=36.1$ (Figure 6f), all landscapes had similar ranges of maximum correlations, whereas we observed case-I relationships in landscape A and case-IV in landscape E (Figure 4f).

There was a decrease in average $\mathrm{F}_{\mathrm{ST}}$ values with increasing MMD at both generation 50 and 500 . The average $\mathrm{F}_{\mathrm{ST}}$ value resulting from a landscape in which the demes were relatively close together (landscape A) was lower than those from landscapes where the demes were relatively far apart at a certain MMD (landscape D and E; see Supplementary Figures 1 and 6).

\section{DISCUSSION}

The results of the present simulation study showed that habitat configuration has a clear influence on patterns of IBD. The rate and likelihood of the appearance of case-I or case-IV $\mathrm{F}_{\mathrm{ST}}$-distance relationships (Hutchison and Templeton, 1999) clearly differed between different landscapes with the same amount of habitat and the same degree of fragmentation and was, thus, affected by habitat configuration and/or deme topology. This result holds true for several types of migration functions (that is, Gaussian and exponential). The common assumption in landscape genetics that patterns of IBD are not influenced by any landscape effects and arise through distanceconstrained migration alone (Spear et al., 2005; Balkenhol et al., 2009; Jenkins et al., 2010) is debatable, as habitat configuration is an intrinsic aspect of landscapes and is a main determinant of deme topology. The maximum correlation between $\mathrm{F}_{\mathrm{ST}}$ and geographic distance or $\mathrm{F}_{\mathrm{ST}} /\left(1-\mathrm{F}_{\mathrm{ST}}\right)$ and $\ln$ (distance) was measured across nearly all pairs of demes in case-I $F_{S T}$-distance plots and at the distance at which the plot flattened in case-IV $\mathrm{F}_{\mathrm{ST}}$-distance plots. The maximum migration distance did not correspond to the distance at which the maximum $\mathrm{F}_{\mathrm{ST}}-$ distance correlation was measured. There is thus a discrepancy between the distance threshold at which the maximum $\mathrm{F}_{\mathrm{ST}}$-distance correlation was measured and the recommended distance threshold for determining landscape effects on gene flow (that is, the maximum migration distance) when the response variable is an estimate of historical gene flow (for example, $\mathrm{F}_{\mathrm{ST}}$ ) and landscape predictor variables are measured directly between demes (as in most landscape genetic studies). Below, we recommend approaches to determine these distance thresholds for both the assessment of the presence and strength of IBD and determining landscape effects on gene flow.

\section{Measuring IBD}

To obtain an indication of the presence of IBD, we recommend landscape geneticists to determine the maximum $\mathrm{F}_{\mathrm{ST}}$-distance correlation (that is, correlation at DMC), by calculating the correlation at a range of maximum distances (Figure 3). Although in the current study the maximum $\mathrm{F}_{\mathrm{ST}}$-distance correlation was measured at different distance thresholds for case-I and case-IV IBD patterns, the correlation coefficients were comparable at both distance thresholds. In some situations, we found $\mathrm{F}_{\mathrm{ST}}$-distance correlations to be absent or negative when calculated over all demes, whereas the maximum correlation calculated over more closely located demes was clearly positive. A lack of an $\mathrm{F}_{\mathrm{ST}}$-distance correlation can, for instance, be interpreted as a complete lack of gene flow between demes or as the absence of distance-limited gene flow (as in the island model). Whether (meta)populations exhibit an IBD pattern (case-I or caseIV) or are completely isolated, is important information for species conservation.

Here we assessed the correlation between $\mathrm{F}_{\mathrm{ST}}$ and geographic distance across all pairs of demes up to a certain threshold distance (that is, DMC). However, in the field of spatial population genetics, IBD is commonly assessed with correlograms that depict the genetic 
a

Generation 50

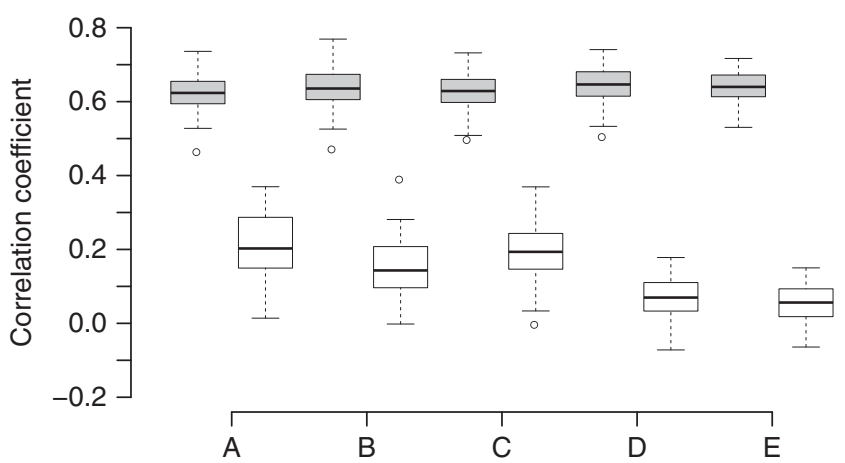

C
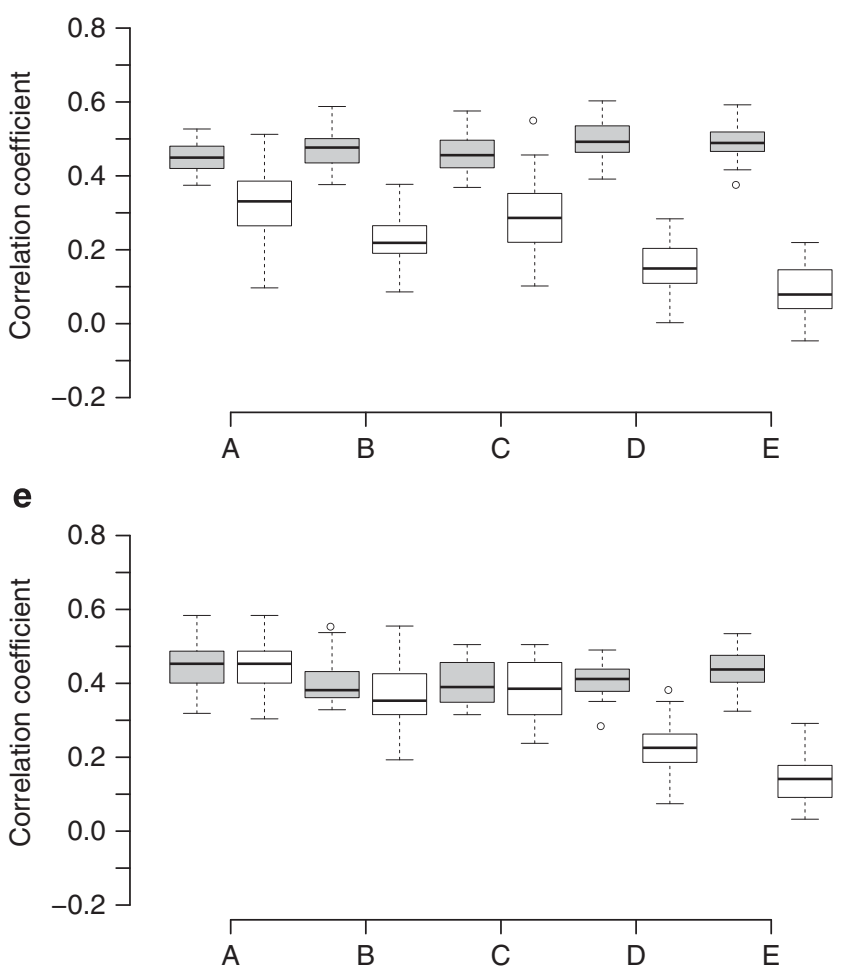

g

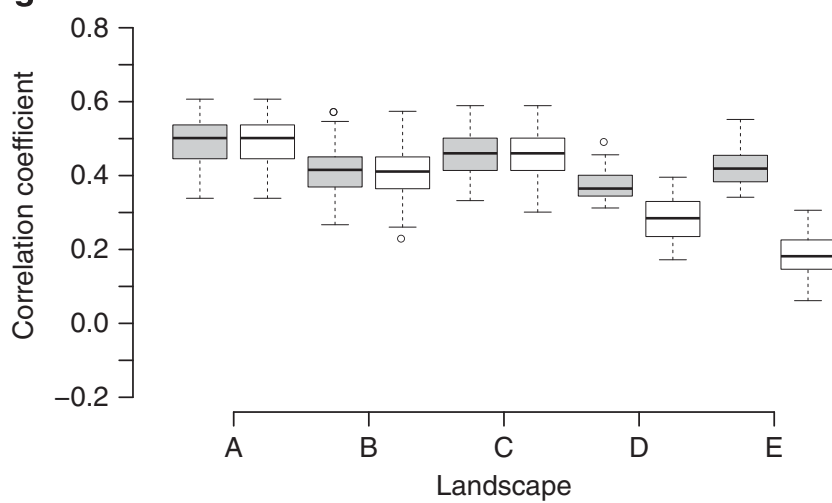

b

Generation 500

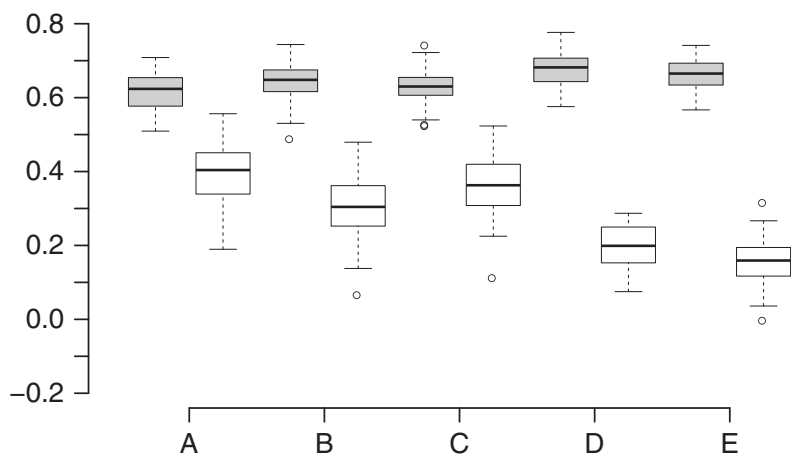

d

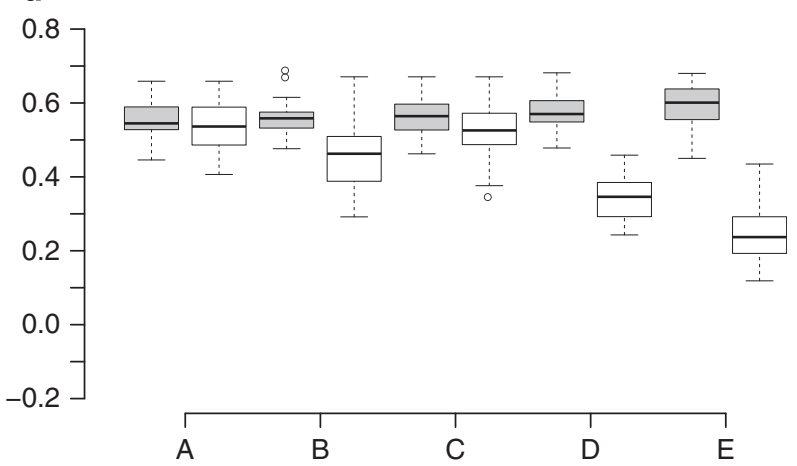

f

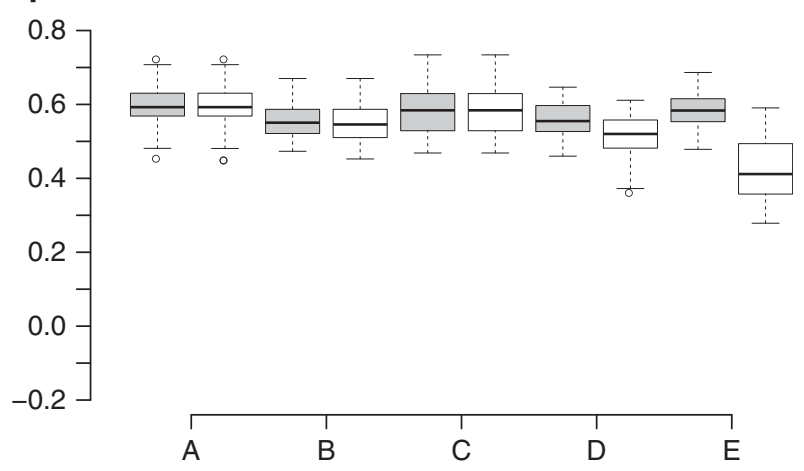

h

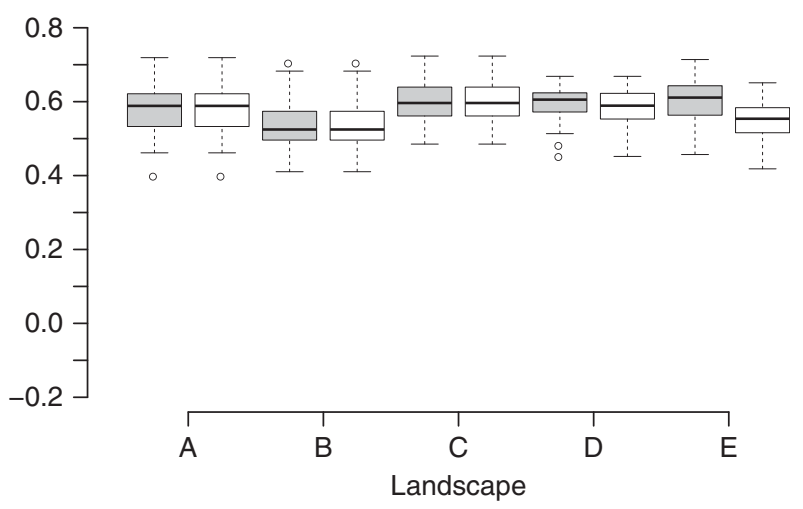

Figure 6 Box plots showing Mantel $r$ correlation coefficient between genetic and geographic distances measured at the distance of DMC (grey plots) and across all deme pairs (white plots) for each of the 50 random deme topologies. Gaussian migration probability functions were used in these simulations. Both plots are depicted for five different neutral landscapes (Figure 2) and a range of MMD (increasing from top to bottom) at generation 50 (a, $\mathbf{c}, \mathbf{e}, \mathbf{g}$ ) and 500 (b, d, f, h). The DMC for the same simulations is shown in Figure 4. 
similarity or differentiation values for different distance lags (for example, distance classes $0-1 \mathrm{~km}, 1-2 \mathrm{~km}, 2-3 \mathrm{~km}$, and so on). Typically Moran's I correlograms are used (Sokal and Wartenberg, 1983; Doligez et al., 1998; Epperson, 2003, 2005), but Mantel correlograms could also be used (Borcard and Legendre, 2012). An advantage of using correlograms for assessing IBD is that it could enhance the comparability of results from empirical landscape genetic studies with those from population genetic theory and simulations. However, empirical (Keller et al., 2013) and simulated (Figure 6) $\mathrm{F}_{\mathrm{ST}}-$ distance plots show that scatter of pairwise $\mathrm{F}_{\mathrm{ST}}$ values is generally high and increases with interdeme distance. In addition, the number of sampled demes is generally low in landscape genetic studies (mean =11; Jenkins et al., 2010). Therefore, in empirical landscape genetic studies, it is likely that the number of samples is too low and the scatter of pairwise $\mathrm{F}_{\mathrm{ST}}$ values too high to get correlograms that do not display erratic fluctuations in Moran's $I$ or Mantel $r$ values, especially in higher distance lags. Such fluctuations would hamper the interpretation of the correlograms.

\section{Measuring landscape effects on gene flow}

The optimal distance threshold to determine landscape effects on gene flow or migration rates should be determined differently to the distance threshold to assess the level of IBD. For such analyses, we support the recommendation of earlier studies (Angelone et al., 2011; Keller et al., 2013) to restrict the analysis to those deme pairs that are within maximum migration distance from each other. This distance can be estimated from, for instance, mark-release-recapture studies (Hassall and Thompson, 2012) or genetic paternity analysis (Kamm et al., 2009). Assuming that the maximum migration distance is determined by physical or behavioural limits of the focal species, intervening landscape should positively or negatively influence migration rates only up to the maximum migration distance. Thus, calculating common interdeme landscape measures, like least cost distances (Rayfield et al., 2010), resistance distances (McRae and Beier, 2007) or quantities of landscape elements within transects (Angelone et al., 2011) between deme pairs that are outside of direct migration distance, would result in landscape information that is irrelevant for migrating individuals and could, thus, bias results. Jaquiéry et al. (2011) used a landscape genetic simulation model and found that landscape effects on gene flow were better detected if only adjacent demes were considered in a regular lattice setup of demes. Thus, in cases where no estimate of the maximum migration distance is available, a more feasible solution could be to only consider neighbouring deme pairs in landscape genetic analysis. In natural settings, where demes are not arranged in regular patterns, neighbouring demes could also be defined by, for instance, Delaunay (Goldberg and Waits, 2010) or Gabriel (Keller et al., 2013) triangulation. The latter has the added benefit that only pairs of demes will be selected that have no intermediate demes and fluctuations in gene flow can thus not be caused by increased (or decreased) gene flow via intermediate demes (Keller et al., 2013). These recommendations are only valid if gene flow is measured over several generations with historical estimates of gene flow such as $\mathrm{F}_{\mathrm{ST}}$. We expect that deme topology will have less effect on measures of contemporary gene flow, stemming for instance from paternity analysis (Kamm et al., 2009) or assignment tests (Manel et al., 2005).

Based on a simple island model, it can be expected that $\mathrm{F}_{\mathrm{ST}}=1$ / $(4 N m+1)$, where $N$ is the effective deme size and $m$ is the immigration rate (Wright, 1931; Whitlock and McCauley, 1999). In accordance with this formula, we found that habitat configurations that resulted in higher average immigration probabilities also resulted in slightly lower average $\mathrm{F}_{\mathrm{ST}}$ values. For the discipline of landscape genetics it is, however, important to realize that these fluctuations in immigration probabilities were caused by deme topology and habitat configuration in the different landscapes and are not analogous to the fluctuations in immigration probability that are either caused by the 'resistance to movement' values of certain landscape elements or nonlinear migration-routes (Spear et al., 2010). The matrix in landscape $\mathrm{E}$ (Figure 2) is a 'barrier' to gene flow because the agents in our model are 'physically incapable' of crossing it (due to very low migration probabilities at long distances) and not because the landscape in the matrix is unfavourable to migration (that is, matrix and habitat had equal migration probabilities in our simulations).

\section{Patterns of IBD}

Hutchison and Templeton (1999) state that 'given sufficient time and stability of conditions, the case-IV pattern should come to resemble more and more a case-I pattern of IBD [...]'. This statement implies that a case-IV state is a non-equilibrium condition that will change to an equilibrium case-I pattern. We can confirm that a case-IV pattern can be an intermediate state between panmixia (that is, our initial condition in the simulations) and a case-I pattern. However, we also found situations in which case-IV patterns appeared to be in a fairly stable state, still apparent after 500 generations. Perhaps, for these cases, it will take many more generations before case-IV relationships change to case-I relationships. Theoretical population genetic studies have shown that also equilibrium situations can produce asymptotic $\mathrm{F}_{\mathrm{ST}}$-distance curves that resemble case-IV patterns. Rousset (1997) determined that, in equilibrium situations, $\mathrm{F}_{\mathrm{ST}} /\left(1-\mathrm{F}_{\mathrm{ST}}\right)$ plotted against log-transformed geographic distance should theoretically produce close to linear correlations for interdeme distance larger than $\sigma$ and smaller than $0.5 \sigma / \sqrt{2 \mu}$, where $\sigma$ is the square root of the variance in the parent-offspring distance and $\mu$ is the mutation rate (Rousset, 1997; Ehrich and Stenseth, 2001). Beyond this distance, the $\mathrm{F}_{\mathrm{ST}}$-distance curve flattens out to form an asymptotic curve. However, given the fact that $\mu=0$ in the present study, we anticipate that our case-IV $\mathrm{F}_{\mathrm{ST}}$-distance relationships were non-equilibrium situations and not asymptotic equilibrium situations (that is, with $\mu=0$ the distance at which an asymptotic equilibrium curve flattens out is expected to be very large). Interestingly, we generally detected similar values of the distance threshold at which we found a maximum correlation between $\mathrm{F}_{\mathrm{ST}}$ and untransformed distances or $\mathrm{F}_{\mathrm{ST}} /\left(1-\mathrm{F}_{\mathrm{ST}}\right)$ and log-transformed distances. Also case-I relationships could result from non-equilibrium situations when demes are sampled from such a small area that even the furthest demes are closer than DMC. It is thus important for landscape geneticists to realize that it is difficult to determine the state of the study system (that is, equilibrium or non-equilibrium) from case-I or case-IV $\mathrm{F}_{\mathrm{ST}}-$ distance relationships. Especially, because the generally great amount of scatter in $\mathrm{F}_{\mathrm{ST}}$-distance plots (Epperson et al., 2010) may make the pattern hard to distinguish and because accurate estimates of $\sigma$ and/or $\mu$ are usually not available in empirical studies (Ehrich and Stenseth, 2001). Nevertheless, more simulation studies should focus on nonequilibrium states of $\mathrm{F}_{\mathrm{ST}}$-distance relationships as these are probably equally important in landscape genetics as equilibrium states (Manel and Holderegger, 2013).

We observed a slightly increasing slope of median $\mathrm{F}_{\mathrm{ST}}$ values in the $\mathrm{F}_{\mathrm{ST}}$-distance plots at larger geographic distances (Figure 5), which might have been caused by some demes that were randomly placed in relatively isolated habitat patches or near the edge of the study area. In our simulation model, these demes got relatively few immigrants from surrounding demes, which probably caused their allele 
frequencies to be more affected by genetic drift leading to higher genetic differentiation between these and other demes. This peripheral population effect has also been documented for many natural (meta)populations (Eckert et al., 2008). Such an effect will not be detectable in population genetic simulation models that use toroidal landscape grids, where the top and bottom as well as the two sides of the study landscape are connected (Doligez et al., 1998) and perhaps also not in those that use a regular lattice setup of finite demes where there are no isolated demes on the edges of the landscape. On the other hand, results from our simulations are only applicable to areas for which it is unlikely that there are many immigrants from outside the study area.

As we were mainly interested in the effects of habitat shape and maximum migration distance on IBD patterns, we kept other variables, like deme size, deme location and number of demes, constant throughout our simulations. However, we acknowledge that spatial or temporal variation in any of these variables would potentially influence the outcome of simulations. Metapopulation dynamics in natural situations (Harrison, 1991) are more complex than the simple model presented in this article. Classical metapopulation theory predicts that extinction and (re)colonization of demes has profound effects on genetic differentiation among demes (Hastings and Harrison, 1994). Fluctuations in population size may not only affect the number of emigrants leaving a deme, but also the rate at which new alleles establish in a deme, which might complicate the interpretation of IBD patterns (Bjorklund et al., 2010).

\section{CONCLUSIONS}

We conclude that the type of IBD pattern that emerges after a certain number of generations is strongly affected by the habitat configuration, deme topology and the maximum migration distance. Therefore, IBD patterns should not be regarded as resulting from only distance-constrained migration, but also from the deme topology and habitat configuration. As the distance at which we measured the highest $\mathrm{F}_{\mathrm{ST}}$-distance correlation did not correspond to the maximum migration distance, we recommend that IBD and landscape effects on gene flow are assessed separately and possibly at different distance thresholds. The rate at which case-I IBD patterns emerge is influenced by an interplay of habitat configuration and maximum migration distance. Landscape geneticists should thus (1) be more aware of the effect of the spatial deme topology on gene flow and (2) of the effect that habitat configuration has on this topology. It may be necessary to (3) assess the presence and intensity of IBD by searching for the maximum $\mathrm{F}_{\mathrm{ST}}$-distance correlation from a subset of deme pairs. The effect of landscape on gene flow can then separately be assessed by (4) using only those pairs of demes that are within migration range of each other. Considering the importance of deme topology on gene flow, (5) more emphasis should be placed on complete sampling of all demes within a study landscape.

\section{DATA ARCHIVING}

Simulation programme, Concordia, is available from the Dryad Digital Repository: doi:10.5061/dryad.m8q30.

\section{CONFLICT OF INTEREST}

The authors declare no conflict of interest.

\section{ACKNOWLEDGEMENTS}

This article forms part of the ENHANCE project, which was funded by the Competence Centre Environment and Sustainability of the ETH Domain. We thank Dave Jenkins, Corey Anderson, the subject editor and one anonymous referee for valuable comments on the manuscript, which greatly improved its contents.

Angelone S, Kienast F, Holderegger R (2011). Where movement happens: scale-dependent landscape effects on genetic differentiation in the European tree frog. Ecography 34 714-722.

Austerlitz F, Dick CW, Dutech C, Klein EK, Oddou-Muratorio S, Smouse PE et al. (2004). Using genetic markers to estimate the pollen dispersal curve. Mol Ecol 13: 937-954.

Balkenhol N, Waits LP, Dezzani RJ (2009). Statistical approaches in landscape genetics: an evaluation of methods for linking landscape and genetic data. Ecography 32 $818-830$

Bjorklund M, Bergek S, Ranta E, Kaitala V (2010). The effect of local population dynamics on patterns of isolation by distance. Ecol Inform 5: 167-172.

Borcard D, Legendre P (2012). Is the Mantel correlogram powerful enough to be useful in ecological analysis? A simulation study. Ecology 93: 1473-1481.

Bowne D, Bowers M (2004). Interpatch movements in spatially structured populations: a literature review. Landscape Ecol 19: 1-20.

Chapman BB, Brönmark C, Nilsson J-Å, Hansson L-A (2011). The ecology and evolution of partial migration. Oikos 120: 1764-1775.

Corney PM, Le Duc MG, Smart SM, Kirby KJ, Bunce RGH, Marrs RH (2004). The effect of landscape-scale environmental drivers on the vegetation composition of British wood lands. Biol Conserv 120: 491-505.

Crispo E, Hendry A (2005). Does time since colonization influence isolation by distance? A meta-analysis. Conserv Genet 6: 665-682.

Doligez A, Baril C, Joly HI (1998). Fine-scale spatial genetic structure with nonuniform distribution of individuals. Genetics 148: 905-920.

Eckert CG, Samis KE, Lougheed SC (2008). Genetic variation across species' geographical ranges: the central-marginal hypothesis and beyond. Mol Ecol 17: 1170-1188.

Ehrich D, Stenseth NC (2001). Genetic structure of Siberian lemmings (Lemmus sibiricus) in a continuous habitat: large patches rather than isolation by distance. Heredity $\mathbf{8 6}$ $716-730$.

Epperson BK (2003). Geographical Genetics. Princeton University Press: Princeton, USA Epperson BK (2005). Estimating dispersal from short distance spatial autocorrelation. Heredity 95: 7-15.

Epperson BK, McRae BH, Scribner K, Cushman SA, Rosenberg MS, Fortin MJ et al. (2010). Utility of computer simulations in landscape genetics. Mol Ecol 19 3549-3564.

Ezard THG, Travis JMJ (2006). The impact of habitat loss and fragmentation on genetic drift and fixation time. Oikos 114: 367-375.

Gardner R, Urban D (2007). Neutral models for testing landscape hypotheses. Landscape Ecol 22: 15-29.

Goldberg CS, Waits LP (2010). Comparative landscape genetics of two pond-breeding amphibian species in a highly modified agricultural landscape. $\mathrm{Mol}$ Ecol 19: 3650-3663.

Goudet J (1995). FSTAT (version 1.2): a computer program to calculate F-statistics. J Hered 86: 485-486.

Guillot G, Leblois R, Coulon A, Frantz AC (2009). Statistical methods in spatial genetics. Mol Ecol 18: 4734-4756.

Hanski I (1998). Metapopulation dynamics. Nature 396: 41-49.

Harrison S (1991). Local extinction in a metapopulation context: an empirical evaluation. Biol J Linnean Soc 42: 73-88.

Hassall C, Thompson DJ (2012). Study design and mark-recapture estimates of dispersal: a case study with the endangered damselfly Coenagrion mercuriale. J Insect Conserv 16: 111-120.

Hastings A, Harrison S (1994). Metapopulation dynamics and genetics. Annu Rev Ecol Syst 25: 167-188.

Holderegger R, Wagner HH (2006). A brief guide to landscape genetics. Landscape Ecol 21: 793-796.

Hubbell SP (2001). The Unified Neutral Theory of Biodiversity and Biogeography. Princeton University Press: Princeton, USA.

Hutchison DW, Templeton AR (1999). Correlation of pairwise genetic and geographic distance measures: inferring the relative influences of gene flow and drift on the distribution of genetic variability. Evolution 53: 1898-1914.

Jaquiéry J, Broquet T, Hirzel AH, Yearsley J, Perrin N (2011). Inferring landscape effects on dispersal from genetic distances: how far can we go? Mol Ecol 20: 692-705.

Jenkins DG, Carey M, Czerniewska J, Fletcher J, Hether T, Jones A et al. (2010). A metaanalysis of isolation by distance: relic or reference standard for landscape genetics? Ecography 33: 315-320.

Kamm U, Rotach P, Gugerli F, Siroky M, Edwards P, Holderegger R (2009). Frequent longdistance gene flow in a rare temperate forest tree (Sorbus domestica) at the landscape scale. Heredity 103: 476-482.

Kampstra P (2008). Beanplot: a boxplot alternative for visual comparison of distributions. J Stat Softw Code Snippets 28: 1-9.

Keller D, Holderegger R, Van Strien MJ (2013). Spatial scale affects landscape genetic analysis of a wetland grasshopper. $\mathrm{Mol}$ Ecol 22: 2467-2482.

Kimura M, Weiss GH (1964). The stepping stone model of population structure and the decrease of genetic correlation with distance. Genetics 49: 561-576.

Manel S, Gaggiotti OE, Waples RS (2005). Assignment methods: matching biological questions techniques with appropriate techniques. Trends Ecol Evol 20 136-142. 
Manel S, Holderegger R (2013). Ten years of landscape genetics. Trends Ecol Evol 28: 614-621.

McRae $\mathrm{BH}$, Beier $\mathrm{P}$ (2007). Circuit theory predicts gene flow in plant and animal populations. Proc Natl Acad Sci USA 104: 19885-19890.

Meineri E, Skarpaas O, Vandvik V (2012). Modeling alpine plant distributions at the landscape scale: do biotic interactions matter? Ecol Model 231: 1-10.

Montgomery DB, Morrison DG (1973). A note on adjusting R². J Financ 28: 1009-1013.

Nei M (1977). F-statistics and analysis of gene diversity in subdivided populations. Ann Hum Genet 41: 225-233.

Paradis E, Baillie SR, Sutherland WJ, Gregory RD (1998). Patterns of natal and breeding dispersal in birds. J Anim Ecol 67: 518-536.

R Development Core Team (2012). R: A Language and Environment for Statistical Computing. R Foundation for Statistical Computing: Vienna, Austria.

Rayfield B, Fortin MJ, Fall A (2010). The sensitivity of least-cost habitat graphs to relative cost surface values. Landscape Ecol 25: 519-532.

Robledo-Arnuncio JJ, Rousset F (2010). Isolation by distance in a continuous population under stochastic demographic fluctuations. J Evol Biol 23: 53-71.

Rousset F (1997). Genetic differentiation and estimation of gene flow from F-statistics under isolation by distance. Genetics 145: 1219-1228.

Slatkin M (1993). Isolation by distance in equilibrium and non-equilibrium populations. Evolution 47: 264-279.
Sokal RR, Wartenberg DE (1983). A test of spatial autocorrelation analysis using an isolation-by-distance model. Genetics 105: 219-237.

Spear SF, Balkenhol N, Fortin MJ, McRae BH, Scribner K (2010). Use of resistance surfaces for landscape genetic studies: considerations for parameterization and analysis. Mol Ecol 19: 3576-3591.

Spear SF, Peterson CR, Matocq MD, Storfer A (2005). Landscape genetics of the blotched tiger salamander (Ambystoma tigrinum melanostictum). Mol Ecol 14: 2553-2564.

Storfer A, Murphy MA, Spear SF, Holderegger R, Waits LP (2010). Landscape genetics: where are we now? Mol Ecol 19: 3496-3514.

Van Strien MJ, Keller D, Holderegger R (2012). A new analytical approach to landscape genetic modelling: least-cost transect analysis and linear mixed models. Mol Ecol 21: 4010-4023.

van Strien MJ, Keller D, Holderegger R, Ghazoul J, Kienast F, Bolliger J (2014). Landscape genetics as a tool for conservation planning: predicting the effects of landscape change on gene flow. Ecol App/ 24: 327-339.

Weir BS, Cockerham CC (1984). Estimating F-statistics for the analysis of populations structure. Evolution 38: 1358-1370.

Whitlock MC, McCauley DE (1999). Indirect measures of gene flow and migration: FST $_{\text {ST }}$ $\neq 1 /(4 \mathrm{Nm}+1)$. Heredity 82: $117-125$.

Wright S (1931). Evolution in Mendelian populations. Genetics 16: 0097-0159.

Wright S (1943). Isolation by distance. Genetics 28: 114-138.

Supplementary Information accompanies this paper on Heredity website (http://www.nature.com/hdy) 